\title{
Erratum: Small RNA biogenesis: Novel roles of an RNase III enzyme
}

Zhaobo Lang and Zhizhong Gong

Nature Plants 2, 16021 (2016); published online 2 March 2016; corrected 14 March 2016

In the version of this News and Views article originally published, 'RPT2' in Fig. 1 should have read 'RTL2'. This has now been corrected. 